\title{
Design and evaluation of an interactive training system for scrub nurses
}

\author{
Bernhard Glaser ${ }^{1}$ - Tobias Schellenberg ${ }^{1}$ - Lucas Koch ${ }^{1}$ - Mathias Hofer ${ }^{2}$. \\ Susanne Modemann ${ }^{3}$. Patrick Dubach ${ }^{1,4}$. Thomas Neumuth ${ }^{1}$
}

Received: 9 September 2015 / Accepted: 28 January 2016 / Published online: 12 February 2016

(C) CARS 2016

\begin{abstract}
Objective The current trend toward increasingly integrated technological support systems and the rise of streamlined processes in the OR have led to a growing demand for personnel with higher levels of training. Although simulation systems are widely used and accepted in surgical training, they are practically non-existent for perioperative nursing, especially scrub nursing. This paper describes and evaluates an interactive OR environment simulation to help train scrub nurses.

Methods A system comprising multiple computers and monitors, including an interactive table and a touchscreen combined with a client-server software solution, was designed to simulate a scrub nurse's workplace. The resulting demonstrator was evaluated under laboratory conditions with a multicenter interview study involving three participating ear, nose, and throat (ENT) departments in Germany and Switzerland.

Results The participant group of 15 scrub nurses had an average of 12.8 years hands-on experience in the OR. A series of 22 questions was used to evaluate various aspects of the demonstrator system and its suitability for training novices. Discussion The system received very positive feedback. The participants stated that familiarization with instrument
\end{abstract}

Bernhard Glaser

bernhard.glaser@medizin.uni-leipzig.de

1 Innovation Center Computer Assisted Surgery (ICCAS), Faculty of Medicine, University of Leipzig,

Semmelweisstr. 14, 04103 Leipzig, Germany

2 ENT Department, Leipzig University Hospital, Leipzig, Germany

3 Acqua Klinik, Leipzig, Germany

4 Department of Otorhinolaryngology, Head and Neck Surgery, Inselspital, University Hospital of Bern, Bern, Switzerland names and learning the instrument table setup were the two most important technical topics for beginners. They found the system useful for acquiring these skills as well as certain non-technical aspects.

Conclusions Interactive training through simulation is a new approach for preparing novice scrub nurses for the challenges at the instrument table in the OR. It can also improve the lifelong training of perioperative personnel. The proposed system is currently unique in its kind. It can be used to train both technical and non-technical skills and, therefore, contributes to patient safety. Moreover, it is not dependent on a specific type of surgical intervention or medical discipline.

Keywords Workflow - Computer-assisted surgery . Computer-assisted instruction · Nursing education · Surgical process model

\section{Introduction}

Improvements in efficiency and workflow in today's operating room (OR) will significantly enhance surgery in the OR of the future [1]. Harnessing technology and redesigning work processes help deliver higher quality at lower cost [2]. Both junior and senior personnel constantly face new challenges as new technologies such as robot-assisted surgery [3] and adaptive workflow systems are introduced [4].

This trend toward increasingly integrated technological support systems and the rise of streamlined processes in the OR have led to the demand for better-trained personnel. Consequently, the traditional apprenticeship model in healthcare no longer meets the needs of learners in perioperative nursing education [5]. Moreover, given the growing shortage of nurses in the workforce, perioperative leaders are 
facing a significant challenge in recruiting new staff who are committed to perioperative nursing [6]. The undersupply of nursing staff is exacerbated by a rise in average age [7]. Furthermore, at a time when there is a critical need for nurses, nursing education is beset by a severe shortage in nursing faculty [8]. New strategies to ensure structured, forward-looking training for novices and to support the lifelong learning of experienced personnel are therefore essential to cope with the predictable need for extended scrub nurse education for tomorrow's OR. Stahl et al. stated that the introduction of new technology into the OR environment can create significant stress despite achieving desirable goals, such as reducing patient flow time, necessitating risk groups among personnel to receive additional support to manage technology transitions [9]. Simulation clearly offers enormous potential for safely developing expertise in procedural skills [10]. The ability to enable personnel to practice and learn in a safe, controlled environment makes simulation a valuable tool for initial training and continued competency verification [11]. Although the need to integrate information technology into undergraduate nursing programs has been recognized, there are few reports of teaching and learning with these new technologies [12].

Currently, interactive training and simulation systems as preparation for the OR almost exclusively target the surgeon's role. Commonly applied to a wide variety of surgical tasks [13] such as robotic laparoscopy [14,15], they are rarely used for OR functions not performed by the surgeon. Sturm et al. analyzed a variety of simulation-based surgical training approaches with skill transfer to the operative setting [16]. Palter and Grantcharov [17] reviewed the simulators available to surgical educators in 2010 and discussed the potential uses of simulation technology in surgery for the assessment of technical and non-technical skills. Although there are several simulation approaches in nursing education, they are rarely used for perioperative nursing and are practically nonexistent for instrument nursing in particular. Most existing approaches focus on the simultaneous training of all parties involved in an intervention. Aggarwal et al. [18] presented a setup for a simulated operating theater project to train and assess the technical and non-technical skills of the entire surgical team. Fort and Fitzgerald [19] described skill learning in a simulated OR with perioperative evidence-based practice modules, such as handling surgical attire (e.g., gloves and hospital gowns) and scrubbing. Sexton et al. [20] conducted team-oriented medical simulations (TOMS) in a full-scale OR in Basel (Switzerland) with complete OR teams, including the scrub nurse.

These simulation scenarios mostly use human-like manikins [21] and rely on human observation to control the progress of the scenario and its evaluation. This requires a great deal of organization and manpower, prepared premises, and expensive equipment. For example, a top-of-the-line, high-fidelity human patient simulator is a large investment [22] and cannot be used for complex surgical interventions. These simulations are definitely justified, especially for surgical crisis management [23] and the development of non-technical skills. However, they are too intricate for the exclusive training of the skills of scrub nurses, especially for beginners lacking technical skills such as knowledge of surgical instrument names and the intervention progress. We had already gained extensive experience with the working environment around the instrument table during our previous work on intra-operative surgical instrument usage detection [24] and scrub nurse viewing [25], which led to the fundamental concept for the creation of a training system. The goal was to focus on the technical skills needed by beginners and personnel changing clinical departments while also supporting the range of scrub practitioners' non-technical skill requirements as systematically identified by Mitchell et al. $[26,27]$.

This article explains the implementation of this concept by describing the architecture and features of a newly developed system called the Nosco Trainer. We evaluate the suitability of this novel system for professional education and the development of technical and non-technical skills.

\section{Methods}

\section{System design: structural system setup}

The Nosco Trainer combines multiple computer systems and monitors to simulate the scrub nurse workplace. Figure 1 provides an overview of the setup and the sensor equipment involved. The heart of the setup comprises a Microsoft PixelSense (formerly called Microsoft Surface) interactive surface-computing platform, henceforth called the instrument table system. This device is based on a Samsung SUR40, which combines a horizontal 40" screen with an integrated computer system, giving it a table-like appearance. Its proportions offer sufficient space for the life-size simulation of an entire typical surgical instrument table. The device can recognize 52 simultaneous multitouch contact points, allowing concurrent interaction on the table with multiple fingers and hands.

The instrument table system has a local area network (LAN) connection to another computer called the operating room simulation system. In the setup used for the study, this system was connected to two monitors. The surgeon screen displayed the scene including the surgeon from the scrub nurse's point of view. Its touchscreen enabled simulated interaction with the surgeon. The second monitor, a conventional screen, displayed the corresponding view of the endoscope. Figure 2 shows both monitors and the complete system in the demonstration OR at ICCAS. 


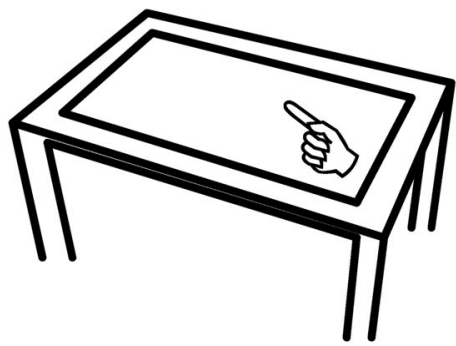

\begin{tabular}{l|l|}
\cline { 2 - 2 } Screens & $\begin{array}{l}\text { Instrument table screen } \\
\text { Samsung SUR40 40" } \\
\text { (Microsoft Pixelsense) } \\
\text { Resolution 1920x1080 } \\
\text { Non-capacitive touch technology with up to } \\
52 \text { simultaneous multitouch points of contact }\end{array}$ \\
& $\begin{array}{l}\text { System embedded in table } \\
\text { Hardware } \\
\text { Windows } 7 \text { Pro 64bit, SP1 } \\
\text { AMD Athlon } 2 \text { X2 245e @ 2.90 Ghz } \\
4 \text { GB Ram }\end{array}$ \\
\cline { 2 - 2 } &
\end{tabular}

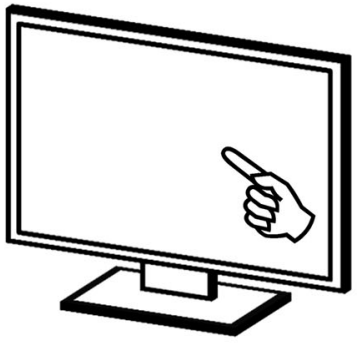

Surgeon screen

Dell Touchscreen 22" (Dell ST2220T)

Resolution $1920 \times 1080$

Capacitive touch technology with up to two simultaneous multitouch points of contact

\section{System}

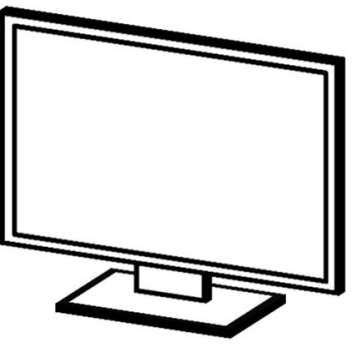

Endoscope screen

Dell TFT 20"

(Dell UltraSharp 2007FP)

Resolution $1600 \times 1200$

No touch capability

Software

Nosco Table Client Software
- Microsoft Visual C\# 2010
- Microsoft Surface 2.0 SDK

Instrument Table System (Client)

\section{ֻัญ}

Communication Interface
OR Simulation System

(Server)

Fig. 1 Overview of the system components

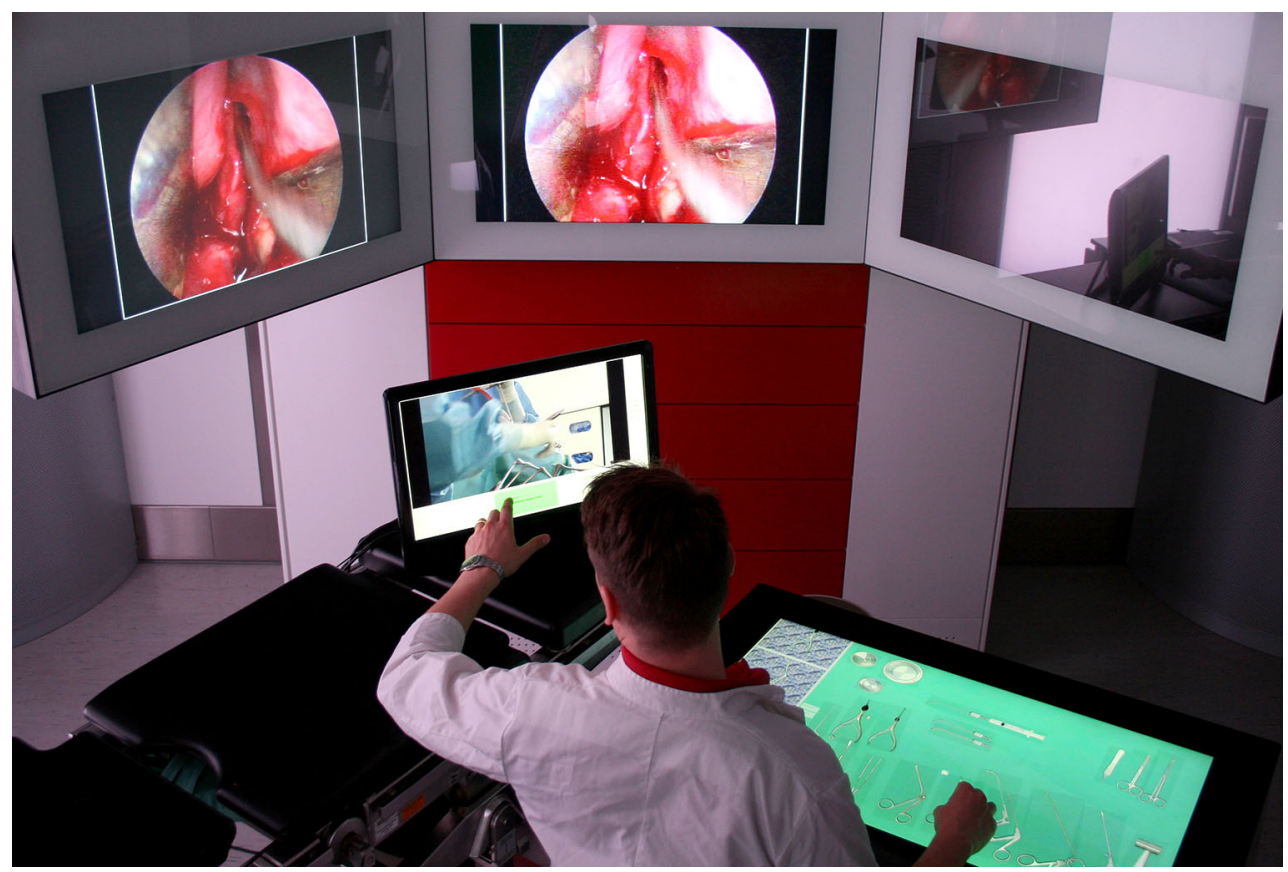

Fig. 2 Demonstration of the trainer system in the demonstration OR at ICCAS. Here, the endoscope screen has been replaced by a connection to the OR monitor matrix 


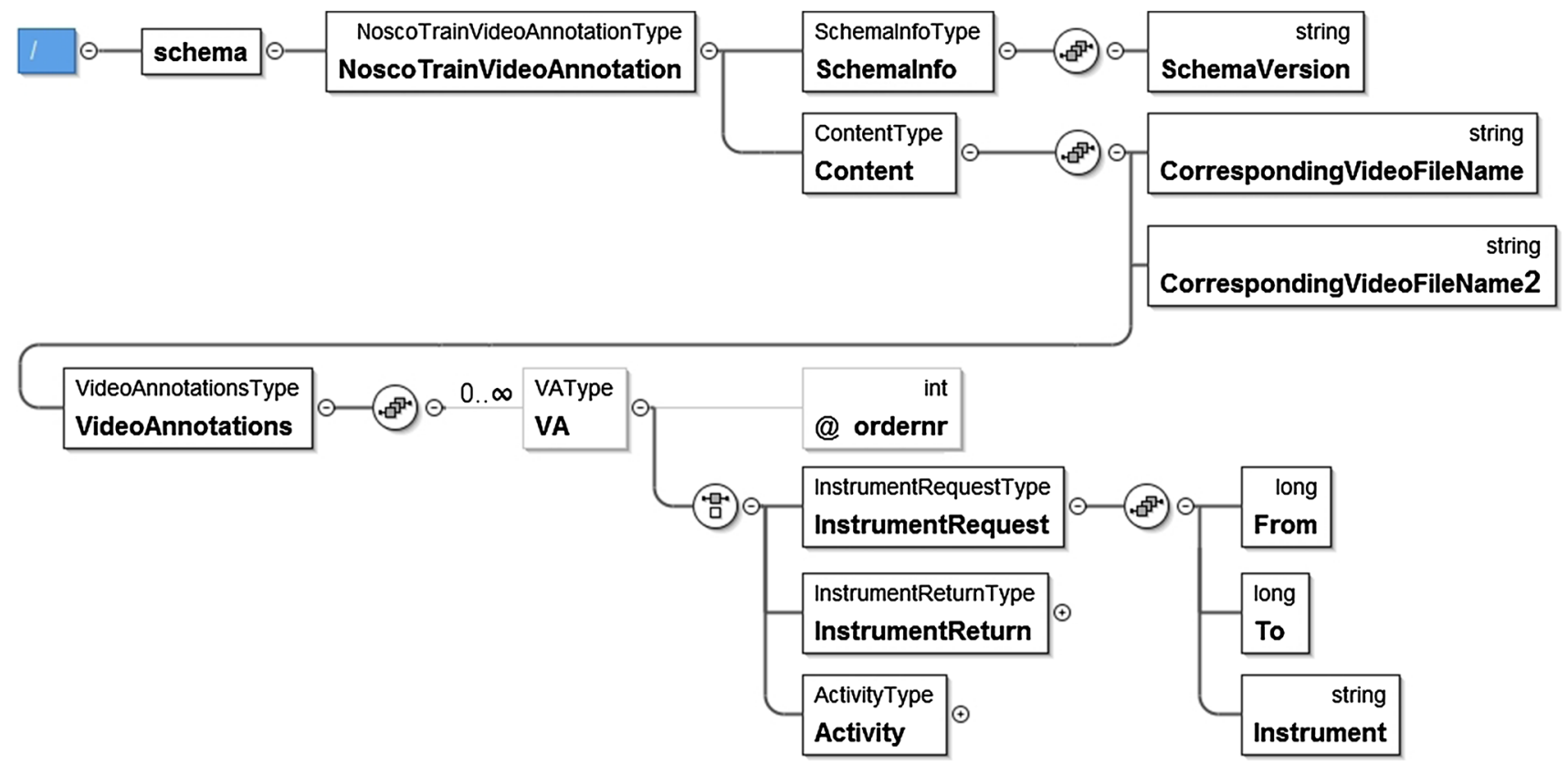

Fig. 3 Logical model view of the nosco.sub XML schema for the annotation of a surgical intervention

\section{System design: dynamic system behavior}

The underlying software framework is based on a clientserver architecture divided into two subsystems. The instrument table system runs on the Nosco Table Client Software written in $C \#$. The choice of programing language was driven by the need to access the hardware features of the PixelSense system, which rely on the Microsoft Surface 2.0 software development kit (SDK) [28]. The client was implemented to be as lean as possible. It handles display and interaction with the instruments and the additional instrument tray. The server can remotely control every aspect displayed on the client. Moreover, it is constantly notified of every change happening on the client, including instrument movement and rotation. The side tray was implemented as a scrollable list displaying slightly downscaled pictures of the instruments on top of the typical underlay mat of a surgical tray. Scrolling is performed by placing a finger on the tray list and dragging it up or down. Instruments can be added and removed by selecting an instrument and pressing one of the arrow buttons next to the tray on the table showing the corresponding direction. Figure 5 shows a screenshot of an instrument table with tray. At any time during the simulation, smooth and simultaneous interaction with the content of the table is possible. Instruments can be moved on the table and also rotated with two fingers. As an additional feature, selecting an instrument causes a small square button to appear for several seconds on the top left corner of its translucent gray selection area. If this button is pressed, a label bearing the name of the instrument briefly appears next to the instrument.
The OR simulation system runs on the Nosco Trainer Server Software written in Java 1.7. Its main function is to handle every aspect of the simulation and all the connected subsystems. The simulation is based on the synchronous playback of different videos and sounds on the surgeon screen and the endoscope screen. A language called nosco.sub was developed in Extensible Markup Language $(X M L)$ to annotate the videos with information relevant to the simulation, as shown in Fig. 3. An upcoming instrument request by the surgeon in the simulation is represented by an element of InstrumentRequestType. The From and To elements represent the beginning and end points in milliseconds for the display of the request for the surgical instrument on the surgeon screen.

During this time, a large button appears under the video on the surgeon screen showing the name of the requested instrument. At the end of the time period, all videos freeze until the participant has passed the correct instrument. The participant can pass an instrument to the virtual surgeon by holding it on the instrument table screen with one hand and simultaneously pressing the button on the surgeon screen. If the wrong instrument is passed or if no or multiple instruments have been selected, the participant is notified by a message window appearing on the surgeon screen. If the correct instrument is passed, the instrument disappears from the instrument table screen and the simulation and all videos continue. The definition of the InstrumentReturnType is analogous to InstrumentRequestType. By pressing the button shown to accept the instrument that the surgeon wants to pass back, it returns to the instrument table screen and the simula- 


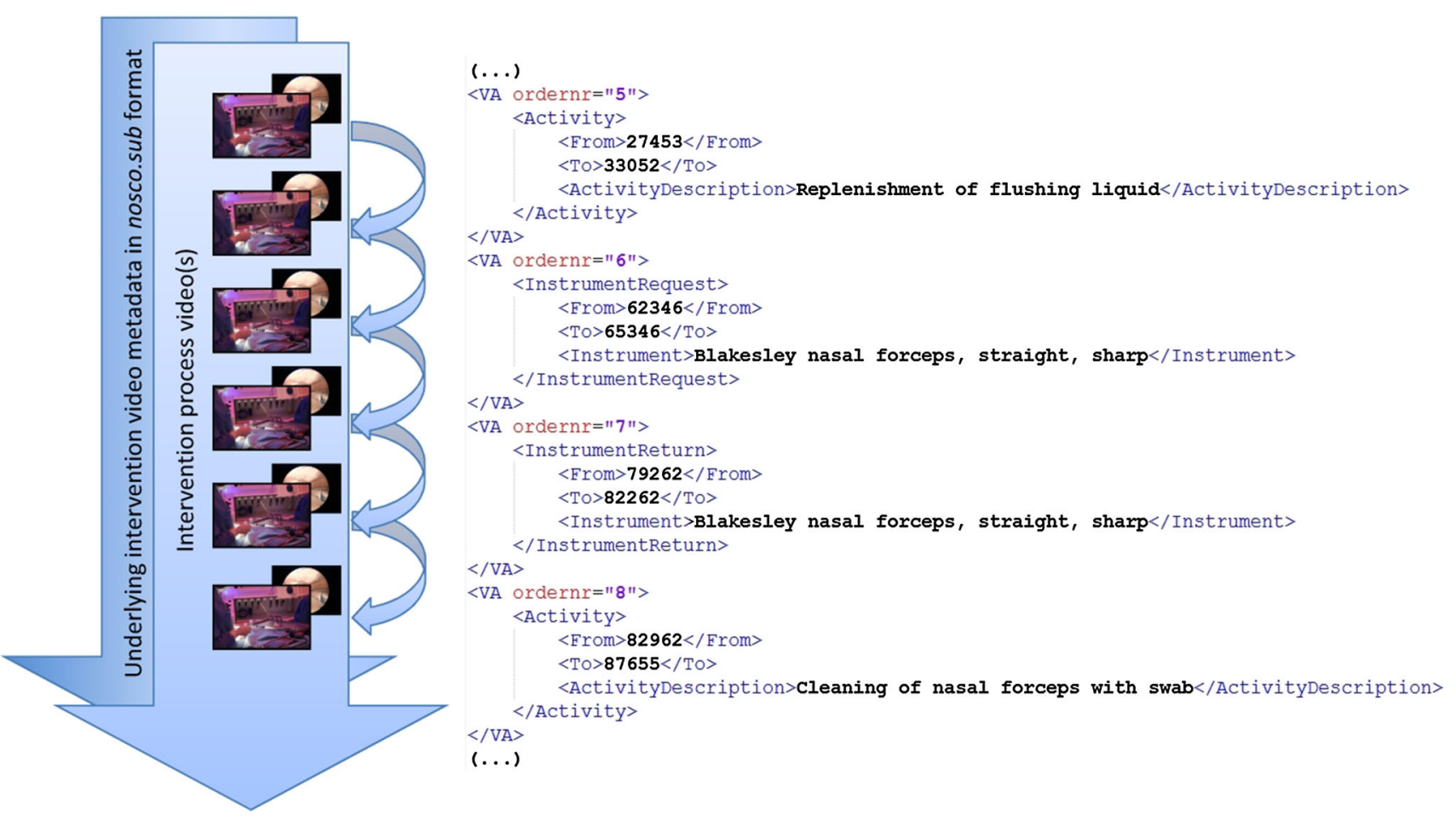

Fig. 4 Excerpt of underlying information video metadata in nosco.sub format

tion continues. ActivityType bundles other possible activities simulated in the trainer. Finally, a custom interview component was integrated for streamlined trainee interviews.

\section{Evaluation study}

\section{Design of the evaluation study}

A multi-center study to evaluate the Nosco Trainer System was conducted under laboratory conditions. Participants were recruited for the study by ENT physicians, who also supervised the medical aspects of the simulation. This study was embedded in a more extensive study which also evaluated aspects of the current level of standardization in the operating room, such as the similarity of surgical instrument descriptions used by scrub nurses [29]. To include a diverse target audience and ensure a significant group of participants, the study was conducted at the ENT departments of three different hospitals: Acqua Klinik in Leipzig, Germany, Inselspital University Hospital of Bern in Switzerland, and Leipzig University Hospital in Germany. The study was conducted in three blocks, one for each hospital, between fall 2014 and spring 2015. Each clinic provided a secluded room where the demonstrator was set up throughout the study. Five participants from each clinic were interviewed, totaling fifteen study participants. A surgical intervention known as functional endoscopic sinus surgery (FESS) was selected as the basis for the study. As described by Stammberger and Posawetz [30], the general aim of FESS is to clear diseased ethmoid clefts and compartments under the guidance of a rigid endoscope and to reestablish ventilation and drainage of the diseased larger sinus via their physiological routes. The intervention included the use of a surgical navigation system. FESS was chosen because of its limited range of instruments (which fit on a single instrument table) and because it is commonly practiced in ENT clinics (to ensure all participants' familiarity with the type of intervention). To give the participants an impression of the simulation environment, a 3-min video recording of the intervention was selected for the simulation. The excerpt was taken shortly after surgical incision when the processus uncinatus is removed, indicating the current stage of the intervention to the participants. The video was annotated with the information to be simulated during the demonstration. Figure 4 shows an excerpt from the associated file created in nosco.sub format.

In total, fourteen instrument requests and responses were contained in the demonstration as well as six activities included solely for information purposes (e.g., cleaning instruments and refilling fluids). These were intended to give the participants an outlook on possible future extensions of the simulation. Figure 5 shows an annotated screenshot of the instrument table at the beginning of the simulation. All necessary instruments were already laid out on the table so that no interaction with the tray was required during the simulation. 


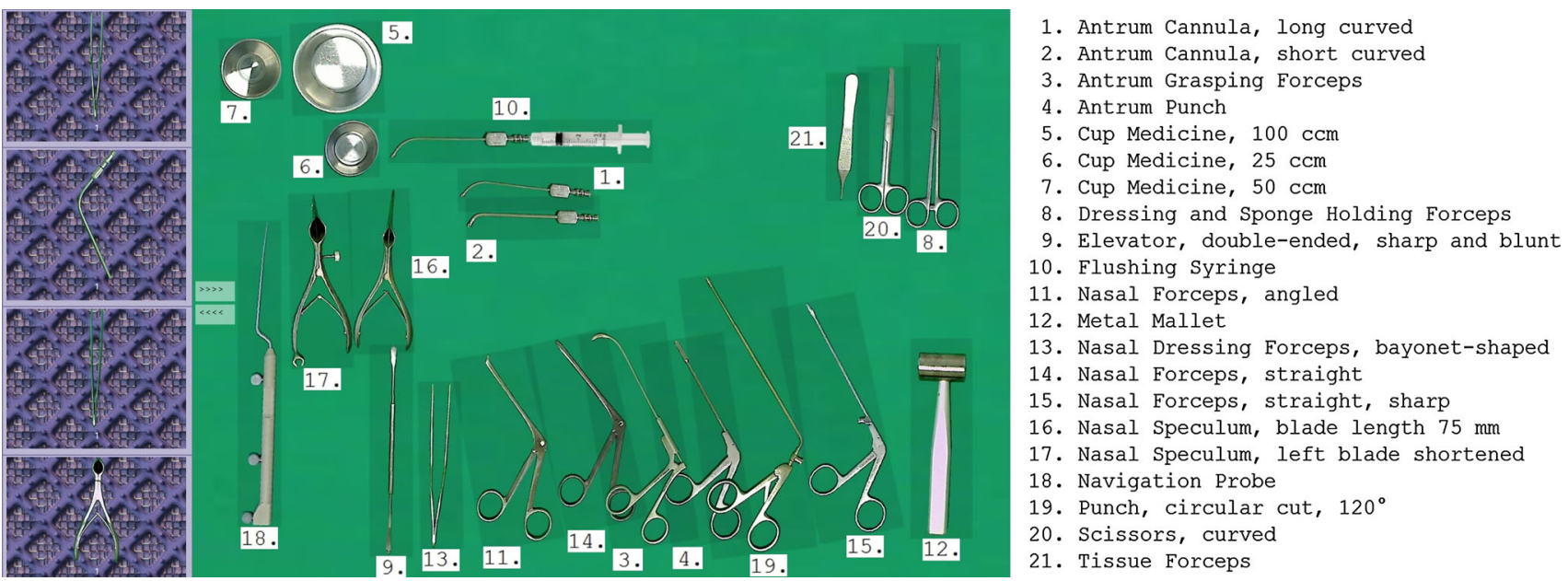

Fig. 5 Left annotated instrument table state at the beginning of the simulation. Right list of instruments visible on the table, sorted alphabetically and without the instruments contained in the additional tray on the left

The participants had already gained hands-on experience of the functionality of the table before the start of the simulation by performing a table setup from scratch, which was not included in this study. The selection of instruments provided on the table was reduced and was not intended to represent any ideal or preferred table state. It included optional spare instruments for potential extension to any intervention, as is the case in actual surgery.

To evaluate the system, a questionnaire was devised to interview the participants before and after performing the demo simulation. All questions were reviewed with experts in the field, both from a medical angle and in terms of user experience. All interviews started with a general set of questions to determine the composition of the participants including factors such as age, language skills, work experience, and experience of training personnel. Subsequent to the demo simulation, the participants were asked twenty-two questions to determine their detailed ratings of various technical and non-technical aspects of the demonstrator system and to ascertain the most important things that beginners could learn with the trainer. For all rating questions, an identical rating scale was chosen ranging from 1 to 5 (1-very good, 2-good, 3-neither good nor bad, 4-poor, 5-bad). All participants took part in the interview voluntarily, were assured anonymity, and were told that they were free not to respond to questions if they wished. They were informed that their performance and speed while working with the system would not be measured, and that the purpose of the demonstration was solely to give them a detailed insight before reviewing and commenting on the demonstration system. For all rating questions, the average rating per clinic was calculated, along with the overall average rating. All questions were translated from German into English, as were all the participants' answers.

\section{Results of the evaluation study}

Two-thirds of the 15 participants were female; one-third was male. Three of the participants were aged between 18 and 30 years, six between 31 and 40, five between 41 and 50, and one between 51 and 60 . Thirteen of the 15 participants were native German speakers, and all participants were fluent in German. All participants were smartphone users. All participants had undergone traditional nursing education with continuing education as an OR specialist with the exception of three participants in the Swiss hospital, who had received equivalent training called qualified operating room technician ${ }^{1}$ or technical operation assistant. ${ }^{2}$ The 15 participants had on average $15.7 \pm 9.4$ years experience as nurses, including $12.8 \pm 9.8$ years as scrub nurses. Eight of the 15 participants had been trained in the same OR in which they were working at the time of the interview. Seven participants had never trained anyone inside the OR before, two participants had trained fewer than 10 persons, and six participants had trained more than 10 persons during their career. All participants had performed surgical instrumentation for the type of intervention presented in the demonstration of the simulator and were familiar with the type of intervention. Table 1 presents the results for the questions for which participants were asked to provide detailed ratings of the basic features of the simulation system (Q1-Q9).

Table 2 presents the results for the block of questions in which participants were asked about their detailed ratings of the effectiveness of the simulator system for training various skills (Q10-Q21).

\footnotetext{
${ }^{1}$ German: Dipl. Fachfrau/-mann Operationstechnik.

${ }^{2}$ German: technische(r) Operationsassistent(in) (TOA).
} 
Table 1 Closed-ended questions regarding the basic features of the simulator system

\begin{tabular}{|c|c|c|c|c|}
\hline Question & Min. & Max. & SD & Total $\varnothing$ \\
\hline How realistic did you find working with the Nosco Trainer? (Q1) & 1.0 & 3.0 & 0.7 & 1.8 \\
\hline How well could you imagine training a complete operation with the Nosco Trainer? (Q2) & 1.0 & 3.0 & 0.7 & 1.7 \\
\hline How easily could you distinguish among the different instruments of the surgical tray? (Q3) & 1.0 & 3.0 & 0.7 & 1.9 \\
\hline How do you rate the reaction speed of the system when moving instruments on the table? (Q4) & 1.0 & 5.0 & 1.0 & 1.9 \\
\hline How do you rate the simulation of the instrument tray on the side? (Q5) & 1.0 & 4.0 & 1.0 & 2.0 \\
\hline How do you rate the simulation of the view of the surgeon? (Q6) & 1.0 & 3.0 & 0.7 & 1.5 \\
\hline How do you rate the simulation of the passing and receiving of instruments? (Q7) & 1.0 & 3.0 & 0.7 & 1.7 \\
\hline How do you rate the sound of the simulation shown? (Q8) & 1.0 & 4.0 & 0.9 & 1.7 \\
\hline How well were you able to follow the operation with the Nosco Trainer? (Q9) & 1.0 & 4.0 & 1.0 & 1.9 \\
\hline
\end{tabular}

The rating scale ranges from 1 (very good) to 5 (bad). The average rating is stated in total including min./max. values and standard deviation

Table 2 Closed-ended questions regarding the effectiveness of the simulator system

\begin{tabular}{|c|c|c|c|c|}
\hline Question & Min. & Max. & SD & Total $\varnothing$ \\
\hline $\begin{array}{l}\text { How suitable is the Nosco Trainer for preparing you } \\
\text { for a new type of operation? (Q10) }\end{array}$ & 1.0 & 3.0 & 0.7 & 1.7 \\
\hline $\begin{array}{l}\text { How suitable is the Nosco Trainer for education and } \\
\text { training? (Q11) }\end{array}$ & 1.0 & 5.0 & 1.0 & 1.7 \\
\hline $\begin{array}{l}\text { How much of an insight into your daily work can } \\
\text { someone who has never orchestrated before gain } \\
\text { with the Nosco Trainer? (Q12) }\end{array}$ & 1.0 & 5.0 & 1.1 & 2.4 \\
\hline $\begin{array}{l}\text { How well does the Nosco Trainer train the ability to } \\
\text { keep track of the situs, the endoscope and the } \\
\text { instrument table at the same time? (Q13) }\end{array}$ & 1.0 & 4.0 & 0.9 & 2.0 \\
\hline $\begin{array}{l}\text { How well does the Nosco Trainer train the ability to } \\
\text { keep your own instrument table in order? (Q14) }\end{array}$ & 1.0 & 5.0 & 1.3 & 2.0 \\
\hline $\begin{array}{l}\text { How well does the Nosco Trainer train the ability to } \\
\text { follow the operation and the workings of the } \\
\text { surgeon? (Q15) }\end{array}$ & 1.0 & 3.0 & 0.8 & 2.0 \\
\hline $\begin{array}{l}\text { How well does the Nosco Trainer train the ability to } \\
\text { know beforehand which instrument the surgeon } \\
\text { will need next? (Q16) }\end{array}$ & 1.0 & 4.0 & 1.0 & 2.5 \\
\hline $\begin{array}{l}\text { How well does the Nosco Trainer train the ability to } \\
\text { change the configuration of the instruments on the } \\
\text { instrument table during the operation? (Q17) }\end{array}$ & 1.0 & 5.0 & 1.1 & 2.0 \\
\hline $\begin{array}{l}\text { How well does the Nosco Trainer train the ability to } \\
\text { spread more calm during real operations? (Q18) }\end{array}$ & 1.0 & 5.0 & 1.3 & 2.5 \\
\hline $\begin{array}{l}\text { How well does the Nosco Trainer train the ability to } \\
\text { make fewer mistakes during long operations? } \\
\text { (Q19) }\end{array}$ & 1.0 & 5.0 & 1.1 & 2.9 \\
\hline $\begin{array}{l}\text { How well does the Nosco Trainer train the ability to } \\
\text { memorize the name and shape of instruments? } \\
\text { (Q20) }\end{array}$ & 1.0 & 3.0 & 0.7 & 1.5 \\
\hline $\begin{array}{l}\text { How well does the Nosco Trainer train the ability to } \\
\text { memorize the designated use of instruments? (Q21) }\end{array}$ & 1.0 & 4.0 & 0.8 & 1.9 \\
\hline
\end{tabular}

The rating scale ranges from 1 (very good) to 5 (bad). The average rating is stated in total including min./max. values and standard deviation
Table 3 presents the answers to the question about the most important things that beginners should learn with the trainer (Q22).

\section{Discussion}

We created a system to simulate a surgical intervention in an OR from the point of view of a scrub nurse by developing 
Table 3 Categorized results for the open-ended question "In your opinion, what is the most important thing that beginners could learn with the Nosco Trainer? (multiple answers permitted)" (Q22), the results show the number of mentions and the percentage rounded to one decimal place

\begin{tabular}{lcc}
\hline Answers given & $\begin{array}{l}\text { Absolute } \\
\text { mentions }\end{array}$ & $\begin{array}{l}\text { Percentage } \\
\text { (rounded) }\end{array}$ \\
\hline Instrument names & 10 & 33.3 \\
Instrument table setup & 7 & 23.3 \\
Process of the intervention & 5 & 16.7 \\
Correct passing of instruments & 4 & 13.3 \\
Order & 3 & 10.0 \\
Speed & 1 & 3.3 \\
\hline
\end{tabular}

client-server software and a connected set of touchscreen monitors and an interactive table. A study was conducted to evaluate both the demonstrator system and its related design decisions.

The system was very positively received with an average of 1.8 rating points for the realism acquired (Q1). All participants said they could imagine training a full-length operation with the system ( $\varnothing 1.7$ for Q2), as long as the intervention was not monotonous. They also agreed that the system was properly suited to prepare them for a new type of operation (Ø 1.7 for Q10) as well as for education and training (Ø 1.7 for Q11). These results are especially promising considering the participants' long mean experience (12.8 years) as scrub nurses. Because all participants were familiar with the type of intervention, any distraction caused by the OR situation presented was minimized. The design decision to use a Microsoft Surface 2.0 to simulate the instrument table proved valuable. The instruments were clearly distinguishable despite lacking a third dimension ( $\varnothing 1.9$ for Q3). Moreover, most participants considered the reaction speed for the movement of instruments on the table to be sufficient ( $\varnothing 1.9$ for Q4). The design decision to simulate the additional tray on the side by using a scrollable list displaying slightly downscaled pictures of the instruments on top of the typical underlay mat of a surgical tray was considered good ( deal of discussion. In general, it was felt that the organization of the tray as a list could be improved, especially because the organized and always identically prepared surgical trays of real ORs minimize the time spent searching for additional instruments. Therefore, the question of whether the system trains the ability to change the instrument table configuration during the operation ( $\varnothing 2.0$ for Q17) received a similar rating and feedback. The same applies to the question of whether the system trains the ability to keep the instrument table in order ( $\varnothing 2.0$ for Q14). Most points of criticism could be resolved by organizing the list differently, e.g., with groups of instrument categories, or by showing the additional tray as a whole, either displayed on the same table after pushing a button or by moving it to a second interactive table. Also, the automatic return of instruments to their original position on the table when returned back from the surgeon needs to be reconsidered.

Showing the scrub nurse's line of sight to the surgeon was well received ( $\varnothing 1.5$ for Q6), as was the implemented mechanism of passing and receiving instruments by pushing a button on the surgeon screen ( $\varnothing 1.7$ for Q7). When developing the system, we compared different possible passing techniques from a man-machine interaction perspective. We chose a two-handed approach (the first on the table touching the chosen instrument and the second pushing the button on the surgeon screen) because it minimized unwanted mistakes such as selecting multiple instruments while still giving the feeling of "handing over something". However, the simulator does not yet cover the skill of correctly passing instruments, which is considered important by the experts (Q21) and a central part of traditional hands-on training. This could be addressed in a future update by replacing the single "pass instrument" button with a selection of photos showing hands holding the instrument, from which the trainee has to choose the right passing technique for the situation.

The audio playback including the surgeon's voice was considered primarily good ( 1.7 for Q8), although the sound quality was often rightfully criticized. Replacing the surgeon with a (possibly virtual) actor with recorded announcements for instrument requests could enhance comprehensibility. This approach could also solve an existing central weakness of the system compared to traditional hands-on training: the fact that unclear verbal statements cannot yet be queried by the trainee. Even so, the participants could easily follow the course of the intervention ( $\varnothing 1.9$ for Q9), although various details led to distractions. For instance, some considered the arrangement of the screens too widely spread or unusual because it did not resemble the familiar environment in their own OR. Also, some participants took longer than others to become acquainted with the user interface. Nevertheless, the question about whether the system could improve the ability to keep track of the different fields of information during an intervention was rated highly ( $\varnothing 2.0$ for Q13), as was the system's ability to train people to follow the operation (Ø 2.0 for Q15). The fact that the course of the intervention and the use of instruments also depend heavily on the individual surgeon was mentioned often and reflected in the question about whether the system trains the ability to recognize the next instrument required ( 2.5 for Q16). However, this may not be a serious issue because the majority of participants felt the trainer was suitable for training the designated use of instruments ( $\varnothing 1.9$ for Q21).

The question about whether the system is able to train the ability to keep calm during real interventions was often answered negatively ( $\varnothing 2.5$ for Q18). The associated question 
about whether the system has positive implications for the scrub nurse's performance during lengthy operations especially revealed the limitations of a simulator (Ø 2.9 for Q19). It became apparent that a simulation would never be able to cover all aspects of the daily routine of a scrub nurse (Ø 2.4 for Q12). Because the evaluation was limited to a small part of a FESS intervention and a group of scrub nurses specialized in ENT surgery, different surgical domains could also require many additional features. It should be borne in mind, though, that the target group consisted of novices who first need to learn basic techniques. With a rating of 1.5 for Q20, the system's ability to teach the names and shapes of instruments was affirmed almost unanimously. Familiarization with instruments' names was the most-cited aspect that beginners ought to learn with the trainer, closely followed by the instrument table setup (Q22).

\section{Conclusions}

Interactive training through simulation is a new approach to prepare novices for the challenges at the instrument table in the OR. It can also improve the lifelong learning of perioperative personnel and provide insights into the fast-growing sector of new technologies and operation methods.

The proposed system is currently unique in its kind, and, for the first time, applies concepts for surgeon training to operating room personnel. Judging by the thoroughly positive feedback and encouragement from the study participants, the new demonstrator was well liked by both surgeons and the OR personnel interviewed. Even criticized parts of the system were met with several proposals for improvement. The results are especially noteworthy because of the very experienced participant group from three ENT departments with a mean value of 12.8 years experience as scrub nurses and with over $50 \%$ of the participants having experience in training personnel. Compared to traditional training, the demonstrator enables lifelike or even critical situations to be simulated without the need for real patients and contributes significantly to patient safety. It can be used to train both technical and certain non-technical skills. Furthermore, the approach circumvents the need for human supervision in every training phase of the learner. Instead, it can adapt to the user's individual self-learning pace. Furthermore, the high cost of real surgical instruments is eliminated. With the hardware costing roughly $€ 10,000$, the system is affordable for training centers and professional schools. The simulation approach presented is not dependent on a specific type of surgical intervention or medical discipline. With an expandable simulation language and an open communication interface, the system is prepared for the future integration of supplementary simulation aspects such as other activities, more monitors, or additional hardware.
A follow-up project will integrate the most promising aspects into an expanded system and then evaluate it with groups of novice scrub nurses in professional schools.

Acknowledgments The authors would like to thank the following people for their kind support and dedication. From the Acqua Klinik and the International Reference and Development Center (IRDC) in Leipzig (Germany): Gero Strauß, Iris Gollnick, and Anja Rothe. From the ENT department at Leipzig University Hospital (Germany): Andreas Dietz and Markus Pirlich. From the Department of Otorhinolaryngology, Head and Neck Surgery, Inselspital, University Hospital of Bern (Switzerland): Marco Caversaccio, Bernd Werle, and Nexhmedin Avdija. From the Institute for Medical Informatics, Statistics and Epidemiology (IMISE) in Leipzig (Germany): Kais Tahar. From the Central Sterilization Department at the University Medical Center of Leipzig (Germany): Kerstin Schröter and her team. From the Innovation Center Computer Assisted Surgery (ICCAS) in Leipzig (Germany): Alexander Oeser, Klemens Birnbaum, Mario Cypko, Christoph Zeumer, Jens Meier, Stefan Franke, Max Rockstroh, Michael Unger, and Richard Bieck. ICCAS is funded by the German Federal Ministry of Education and Research (BMBF), the Saxon Ministry of Science and Fine Arts (SMWK) in the Unternehmen Region with grant number 03Z1LN12, the European Regional Development Fund (ERDF), and the government of Saxony under measures to support the technology sector.

\section{Compliance with ethical standards}

Conflict of interest The authors declare that they have no conflict of interest.

Ethical standards This article does not contain patient data.

\section{References}

1. Cleary K, Kinsella A, Mun SK (2005) OR 2020 workshop report: operating room of the future. International Congress series, vol 1281, pp 832-838

2. Archer T, Macario A (2006) The drive for operating room efficiency will increase quality of patient care. Curr Opin Anesthesiol 19(2):171-176

3. Barbash GI, Glied SA (2010) New technology and health care costs-the case of robot-assisted surgery. N Engl J Med 363(8):701-704

4. Sutherland J, Van den Heuvel W (2006) Towards an intelligent hospital environment: adaptive workflow in the OR of the future. In: Proceedings of the 39th annual Hawaii international conference on system sciences, 2006. HICSS'06, vol 5, p 100b

5. Granger J, Hebb T, Lavallee R, Murray M (2011) Team training simulation in perioperative nursing education. Can Oper Room Nurs J 29(2):6-8, 10-11, 14 passim

6. Trice LB, Brandvold C, Bruno E (2007) Practice and education: partnering to address the perioperative nursing shortage. AORN J 86(2):259-264

7. Chang EM, Hancock KM, Johnson A, Daly J, Jackson D (2005) Role stress in nurses: review of related factors and strategies for moving forward. Nurs Health Sci 7(1):57-65

8. Sanford PG (2010) Simulation in nursing education: a review of the research. Qual Rep 15(4):1006-1011

9. Stahl JE, Egan MT, Goldman JM, Tenney D, Wiklund RA, Sandberg WS, Gazelle S, Rattner DW (2005) Introducing new technology into the operating room: measuring the impact on job performance and satisfaction. Surgery 137(5):518-526 
10. Kneebone R (2005) Evaluating clinical simulations for learning procedural skills: a theory-based approach. Acad Med 80(6):549553

11. Flurry M, Brooke S, Micholetti B, Natoli N, Moyer K, Mnich S, Potochny J (2012) Nurse training with simulation: an innovative approach to teach complex microsurgery patient care. Ann Plast Surg 69(4):459-461

12. Kenny A (2002) Online learning: enhancing nurse education? J Adv Nurs 38(2):127-135

13. Sutherland LM, Middleton PF, Anthony A, Hamdorf J, Cregan P, Scott D, Maddern GJ (2006) Surgical simulation: a systematic review. Ann Surg 243(3):291

14. Hanly EJ, Marohn MR, Bachman SL, Talamini MA, Hacker SO, Howard RS, Schenkman NS (2004) Multiservice laparoscopic surgical training using the da Vinci surgical system. Am J Surg 187(2):309-315

15. Lerner MA, Ayalew M, Peine WJ, Sundaram CP (2010) Does training on a virtual reality robotic simulator improve performance on the da Vinci surgical system? J Endourol 24(3):467-472

16. Sturm LP, Windsor JA, Cosman PH, Cregan P, Hewett PJ, Maddern GJ (2008) A systematic review of skills transfer after surgical simulation training. Ann Surg 248(2):166-179

17. Palter VN, Grantcharov TP (2010) Simulation in surgical education. CMAJ Can Med Assoc J 182(11):1191-1196

18. Aggarwal R, Undre S, Moorthy K, Vincent C, Darzi A (2004) The simulated operating theatre: comprehensive training for surgical teams. Qual Saf Health Care 13(suppl 1):i27-i32

19. Fort C, Fitzgerald B (2011) How simulation improves perioperative nursing. Nurse 2014 5(2):36-42

20. Sexton B, Marsch S, Helmreich R, Betzendoerfer D, Kocher T, Scheidegger D, TOMS team (1998) Participant evaluation of team oriented medical simulation. In: Henson LC, Lee AC (eds) Simulators in anesthesiology education. Springer, New York, pp 109-110

21. Grenvik A, Schaefer J (2004) From Resusci-Anne to Sim-Man: the evolution of simulators in medicine. Crit Care Med 32(2):S56-S57
22. Rauen CA (2004) Simulation as a teaching strategy for nursing education and orientation in cardiac surgery. Crit Care Nurse 24(3):46-51

23. Moorthy K, Munz Y, Forrest D, Pandey V, Undre S, Vincent C, Darzi A (2006) Surgical crisis management skills training and assessment: a stimulation-based approach to enhancing operating room performance. Ann Surg 244(1):139-147

24. Glaser B, Dänzer S, Neumuth T (2014) Intra-operative surgical instrument usage detection on a multi-sensor table. Int Comput Assist Radiol Surg 10(3):351-362

25. Glaser B, Koch L, Schellenberg T, Neumuth T (2014) Eye-tracking analysis of scrub nurse viewing behavior. In: Proceedings of the 5 th workshop on modeling and monitoring of computer assisted interventions (M2CAI)

26. Mitchell L, Flin R (2008) Non-technical skills of the operating theatre scrub nurse: literature review. J Adv Nurs 63(1):15-24

27. Mitchell L, Flin R, Yule S, Mitchell J, Coutts K, Youngson G (2011) Thinking ahead of the surgeon. An interview study to identify scrub nurses' non-technical skills. Int J Nurs Stud 48(7):818-828

28. Microsoft ${ }^{\circledR}$ Surface ${ }^{\circledR}$ 2.0 SDK and Runtime (updated 2 Feb 2012). http://www.microsoft.com/en-us/download/details.aspx? $\mathrm{id}=26716$. Accessed 09 Apr 2015

29. Glaser B, Schellenberg T, Koch L, Hofer M, Modemann S, Dubach P, Neumuth T (2015) Not these scissors, the other scissors. A multicenter study comparing surgical instrument descriptions used by scrub nurses. In: Proceedings of iOR15: 1st international workshop on intelligent operating rooms, 17th international conference on E-health networking, application and services (IEEE Healthcom 2015), Boston, pp 21-25

30. Stammberger H, Posawetz W (1990) Functional endoscopic sinus surgery-concept, indications and results of the Messerklinger technique. Eur Arch Otorhinolaryngol 247(2):63-76 